\title{
Assessment of Groundwater Vulnerability using Vulnerability Index (Static Water Level, Cation Exchange Capacity, Organic Contents, Nitrate and Chloride) (SCONC) in Part of Mamfe Embayment South Eastern Nigeria
}

\author{
${ }^{1}$ A.N. Ugbaja, ${ }^{2}$ U.A. Ugbaja, ${ }^{2}$ A.E. Bassey, ${ }^{1}$ E.A. Amah and ${ }^{2}$ O.E. Offiong \\ ${ }^{1}$ Department of Geology, \\ ${ }^{2}$ Department of Pure and Applied Chemistry, University of Calabar, Calabar, Nigeria
}

Correspondence

A.N. Ugbaja, Department of Geology,

University of Calabar, Calabar,

Nigeria

Received: April 26, 2018

Accepted: July 12, 2018

Published online: November 25, 2020

\begin{abstract}
The following form the focus of the research work: Determine the physical parameters like conductivity, color, dissolved oxygen, $\mathrm{pH}$ and temperature, chemical and biological parameters. Using static water level, cation exchange capacity, organic contents, nitrate and chloride to evaluate the vulnerability of the aquifers in the study area. Eighty-eight water samples were collected from 22 locations across the periods of dry (January), dry-wet (March), wet (June), wet-dry (September), to highlight the influence of micro-climatic conditions between January to September 2015 on groundwater vulnerability. In this study, the relationship between various elements has been studied using correlation analysis, cluster analysis, factor analysis and vulnerability index (using the Static water level, Cation Exchange Capacity, Organic Contents, Nitrate and Chloride). Vulnerability index reveals that intrusive and Eze-Aku Formation are more vulnerable than Asu-River Group. The data also show that the aquifer in the study area are not vulnerable to nitrate, organic content and CEC but are rather vulnerable to fecal coliform due to water from runoff that infiltrates into the aquifer from the vadose zone during precipitation in the area.
\end{abstract}

Keywords: Cross river state, groundwater, Mamfe embayment, Nigeria, vulnerability index (SCONC)

\section{INTRODUCTION}

In Cross River State, the Mamfe Embayment is the least studied in terms of groundwater quality/potentials.
The area has high potentials, where a lot geochemical study was carried out with drilling. Despite this, nothing has been done on the quality including the vulnerability of the aquifer to surface contamination. In assessing the

C) The Author(s) 2020. This article is licensed under a Creative Commons Attribution 4.0 International License, which permits use, sharing, adaptation, distribution and reproduction in any medium or format, as long as you give appropriate credit to the original author(s) and the source, provide a link to the Creative Commons license, and indicate if changes were made. The images or other third-party material in this article are included in the article's Creative Commons license, unless indicated otherwise in a credit line to the material. If material is not included in the article's Creative Commons license and your intended use is not permitted by statutory regulation or exceeds the permitted use, you will need to obtain permission directly from the copyright holder. To view a copy of this license, visit http://creativecommons.org/ licenses/by/4.0/. 


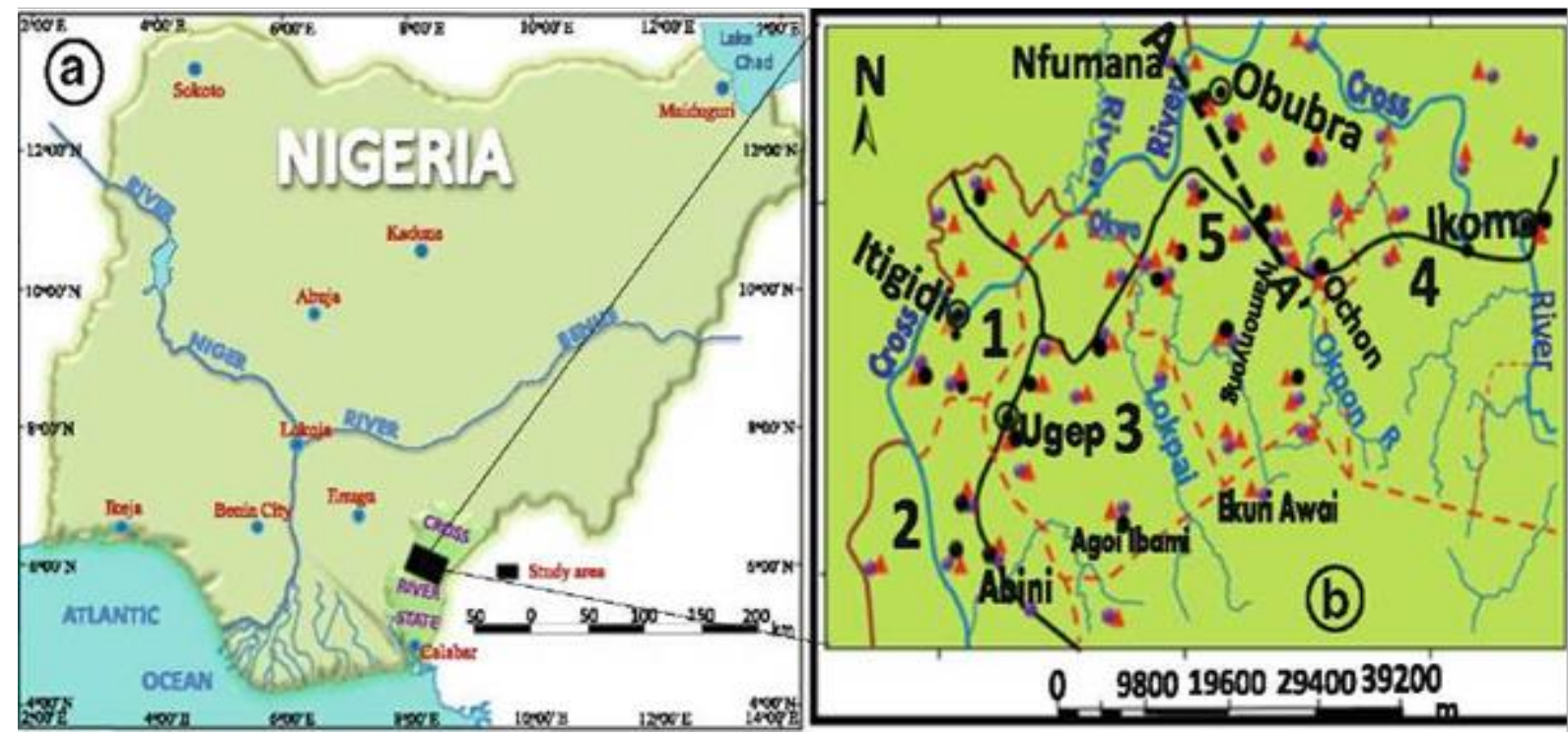

Fig. 1: Map of the studied area showing samples locations/drainage pattern

quality of groundwater in a heterogeneous area like the study area, it is imperative to understand the geochemical circulation of groundwater bearing in mind the different lithologic units. Ground-water vulnerability to contamination was defined by the National Research Council (1993) as the tendency or likelihood for contaminants to reach a specified position in the groundwater system after introduction at some location above the uppermost aquifer based on whether the assessment was contaminant specific, if so, is "specific vulnerability," if the contaminant is general, "intrinsic vulnerability." is apply. Ground-water vulnerability is a function not only of the properties of the ground-waterflow system (intrinsic susceptibility) but also of the proximity of contaminant sources, characteristics of the contaminant and other factors that could potentially increase loads of specified contaminants to the aquifer and (or) their eventual delivery to a ground-water resource (Vowinkel et al., 1996). The geochemical properties of groundwater also depend on the chemistry of water in the recharge area as well as the different geochemical processes that are occurring in the subsurface. These geochemical processes are responsible for the seasonal and spatial variations in groundwater chemistry (Matthess, 1982). Generally, groundwater at the discharge zones tend to have higher mineral concentration compared to that at the recharge zones due to the longer residence time and prolonged contact with the aquifer matrix (Freeze and Cherry, 1979). Further, the weathering of primary and secondary minerals also contributes to cations and silica in the system (Jacks, 1973; Bartarya, 1993). The vulnerability potential of an aquifer to groundwater contamination is to a large extent a function of the susceptibility of its recharge area to infiltration. Areas that are replenished at a high rate are generally more vulnerable to contaminant than those replenished at a slower rate. Groundwater pollution is caused by a variety of substances originating from many different activities. Most of the contaminants that commonly cause concern originate above ground, often as the result of human activities. After release at the land surface, the contaminant may infiltrate downward through the soil, vadose zone and saturated zone finally, reaching the aquifer. Soil overlying the water table provides the primary protection against groundwater pollution, because, sediment and other insoluble forms of contamination become trapped within the soil pores. Some chemical are absorbed or react chemically with various soil constituents, thereby preventing or slowing the migration of these pollutants into the ground waters. In addition plants and soil microorganism use some potential pollutants such as nitrogen, as nutrients for growth, thereby depleting the amount that reaches the groundwater (Hearne et al., 1992).

Study area: The study area lies between latitudes $5^{\circ} 15^{\prime}$ and $6^{\circ} 15^{\prime} \mathrm{N}$ and longitudes $7^{\circ} 45^{\prime}$ and $8^{\circ} 45^{\prime} \mathrm{E}$ (Fig. 1). It is located within the sub-equatorial climatic region of Nigeria with a total annual rainfall of more than 300 to $400 \mathrm{~cm}$. The temperature ranges from 25 to $28^{\circ} \mathrm{C}$. The area experiences two seasons, these are the wet season which lasts from April to September with a peak in June and July while the dry seasons lasts from October to March (Iloeje, 1991). The elevation of the study area ranges from 14 to $170 \mathrm{~m}$ above sea level. The relief is characterized by undulations running in an undefined direction and variably demarcating the very lowland 


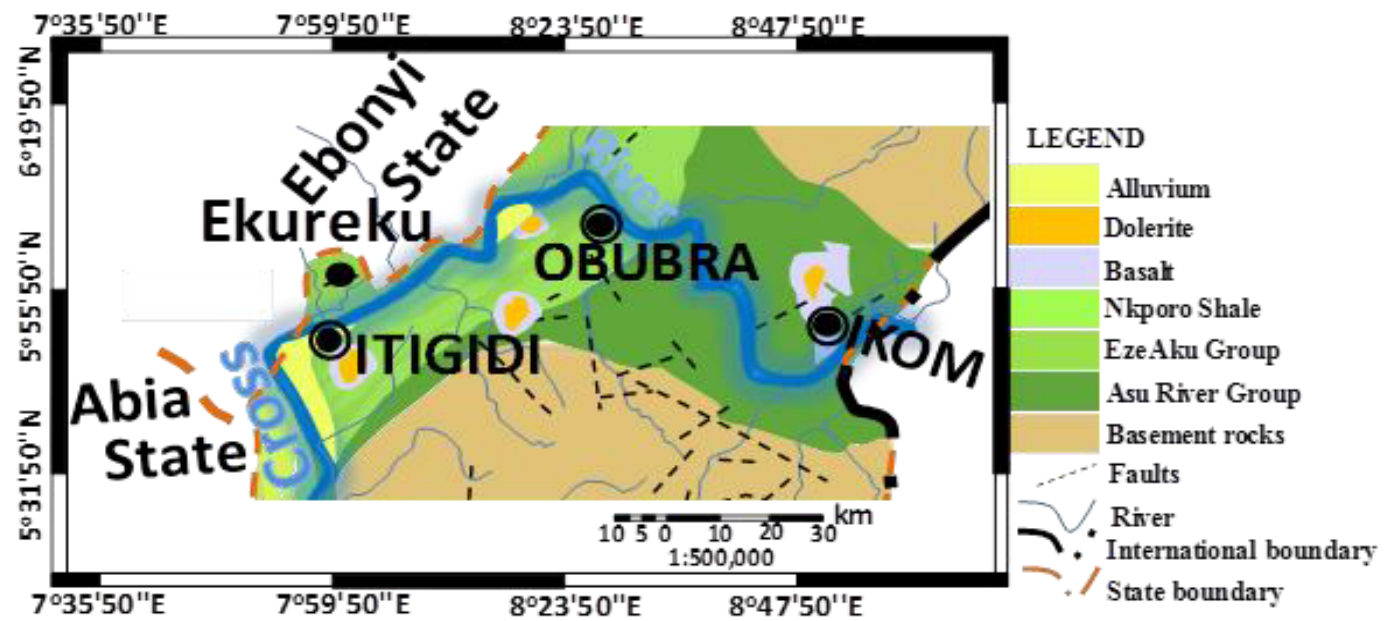

Fig. 2: Geologic map of the study area

areas from moderate relief landmarks. The occurrence of the low plains is occasionally broken by inselbergs of granite, basalts in the southern portion of the study area. In the sediment-filled portions, the low plains are occasionally broken by flat-topped hills of sandstone ridges and igneous intrusive with highly ferroginized sandstones with gravels resulting from uplifts. The area is drained by the Cross River with major tributaries like Udip, Ukong, Lakpoi, Okwo and Okpon rivers (Fig. 1). These rivers form a network of a dendritic drainage system. The minor rivers and or streams that feed these major ones are, however, perennial and trace their origin from the Enugu escarpment and the Cameroon highlands.

Geology: The study area is underlain by two major lithologic units: Crystalline basement and Cretaceous sediments. The crystalline basement rocks occupy the extreme south of the study area. Also, there are intermediate rocks scattered in patches around Obubra, Iyamayong, Iyamitet, Ikom, Nkpani and Usumutong (Fig. 2). The Cretaceous sediments cover about $90 \%$ of the study area. Asu River Group is the basal and oldest recorded sediment in the study area. It is dominated by bluish-gray/black to olivine brown shale and sandy shale, fine-grained micaceous calcareous sandstone and siltstone with limestone lenses. The shale is often carbonaceous and pyritic which indicates that the sediments were deposited under a poorly oxygenated shallow water environment of restricted circulation, an indication of low energy environment (Petters et al., 1987).

Hydrogeology: In basement provinces, groundwater occurrence depends exclusively on discontinuities like fractures, joints, fissures and weathered lithozones. The fissures of crystalline rocks are limited to shallow depths and water movement is lateral in the direction of the gradient downwards to the drainage area. Fracturing and fissuring is a common phenomenon in basalts because of the tectonic chilling effects on them, which develops fractures. About $60 \%$ of groundwater is habited in weathered-fresh bedrock transition with aquifer yields of 0.2-3.5 L/sec. (CRBDA (Cross River Basin Development Authority), 1982). According to Petters (1989) recharge to the weathered zones and joints system is greatly retarded in significantly lateritic cover areas. This is attributed to the high content of the impermeable clay in the laterite. CRBDA (Cross River Basin Development Authority) (1982) put the yield for this province (weathered zones) at $84.4-345.6 \mathrm{~m}^{3} / \mathrm{day}$. Static Water Level (SWL) is between 4.6-19.8 $\mathrm{m}$ in Obubra and 12.2-21.4 $\mathrm{m}$ for part of Ikom in the study area. Boreholes depths range between $25-47 \mathrm{~m}$ in the study area. Shale-sandstone or shale/siltstone province is the largest hydrogeological province in the study area, occupying about $80 \%$ of the study area. This area cuts across locations like Obubra, Apiapum, Nko, Ekori, Ugep, Ochom and Agara Ekureku. It constitutes the geologic Asu River Group and Eze-Aku formation. These sediments are slightly folded, tilted and at times broken by faults. Fractures, fissures and joints commonly occur in sandstones and sandstone affiliated sediments, but are commonly restricted to shallow depths of $20-50 \mathrm{~m}$. Permeability of the study area is influenced by the nature and texture of the sediment type, constituting the study area. For example permeability is moderate in porous, fissured and fractured sandstone/Shale but very low in impervious shale and siltstones. Shale/siltstones province record very low aquifer yield of $0.05-0.5 \mathrm{~L} / \mathrm{sec}$, while some sub-area record up to $2.02 \mathrm{~L} / \mathrm{sec}$ (CRBDA, 1982). 


\section{MATERIALS AND METHODS}

Sampling: Water samples were collected from various locations within the study area (Table 1). The groundwater samples were collected to cover (4) microclimatic condition (Table 2) using pre-washed two-liter polythene plastic bottles for chemical and biological analysis while $250 \mathrm{~mL}$ polythene plastic was used to collect samples for heavy metals. The samples

Table 1: Sample location and type of sample

\begin{tabular}{|c|c|c|c|c|c|c|}
\hline Location name & Latitude & Longitude & Northing & Easting & Geology (lithology) & Type of sample \\
\hline Ikom & $5^{\circ} 56.805$ & $8^{\circ} 43.343$ & 657160 & 469113 & Basalt & $\mathrm{Gw}$, \\
\hline Ikom & $5^{\circ} 57.123$ & $8^{\circ} 43.260$ & 657747 & 468960 & Basalt & Gw, soil \\
\hline Ochon stream & $5^{\circ} 55.759$ & $8^{\circ} 26.659$ & 655257 & 438334 & Sandstone & SW \\
\hline Ochon & $5^{\circ} 55.656$ & $8^{\circ} 26.567$ & 655066 & 438165 & Sandstone/shale & Gw \\
\hline Edondon & $5^{\circ} 51.922$ & $8^{\circ} 25.608$ & 648185 & 436385 & Sandstone & Gw, soil \\
\hline Iyamoyong & $5^{\circ} 57.950$ & $8^{\circ} 21.164$ & 659304 & 428200 & Basalt/shale & $\mathrm{Gw}$ \\
\hline Iyamitet & $5^{\circ} 52.889$ & $8^{\circ} 20.130$ & 649981 & 426280 & Shale/siltstone & Gw \\
\hline Obubra & $6^{\circ} 04.092$ & $8^{\circ} 20.379$ & 670623 & 426766 & Basalt/shale & Gw, soil \\
\hline Apiapum & $5^{\circ} 59.806$ & $8^{\circ} 16.985$ & 662734 & 420493 & shale/siltstone & Gw \\
\hline Ofudua & $5^{\circ} 58.539$ & $8^{\circ} 15.508$ & 660402 & 417769 & Siltstone/shale & Gw \\
\hline Ababene & $5^{\circ} 57.208$ & $8^{\circ} 15.654$ & 657949 & 418035 & Silt/sandstone & Gw \\
\hline Onyadama & $5^{\circ} 56.281$ & $8^{\circ} 14.492$ & 656245 & 415889 & Silt/sandstone & Gw, soil \\
\hline Nko & $5^{\circ} 52.281$ & $8^{\circ} 11.238$ & 648883 & 409875 & Silt/sandstone & Gw, soil \\
\hline Ekpenti & $5^{\circ} 53.287$ & $8^{\circ} 07.356$ & 650747 & 402714 & Silt/sandstone & Gw \\
\hline Ekori & $5^{\circ} 52.381$ & $8^{\circ} 07.165$ & 649078 & 402361 & Silt/sandstone & Gw \\
\hline Ugep & $5^{\circ} 48.252$ & $8^{\circ} 04.736$ & 641476 & 397864 & Silt/sandstone & Gw, soil \\
\hline Ebom & $5^{\circ} 50.803$ & $8^{\circ} 58.194$ & 646197 & 385799 & Sandstone & Gw \\
\hline Usumutong & $5^{\circ} 49.847$ & $8^{\circ} 01.074$ & 644430 & 391113 & Basalt/sandstone & Gw \\
\hline Adim & $5^{\circ} 43.796$ & $8^{\circ} 02.494$ & 633272 & 393715 & Shale/siltstone & Gw, soil \\
\hline Agwugune & $5^{\circ} 40.929$ & $8^{\circ} 00.719$ & 627997 & 390428 & Shale/siltstone & Gw \\
\hline Abini & $5^{\circ} 41.325$ & $8^{\circ} 03.860$ & 628711 & 396209 & Siltstone & Gw \\
\hline Igbo Ekureku (Agbara) & $5^{\circ} 59.283$ & $8^{\circ} 01.266$ & 661813 & 391497 & Sandstone & Gw \\
\hline
\end{tabular}

Gw: Groundwater; sw: surface water

Table 2: The groundwater water samples were collected to cover 4 microclimatic conditions

\begin{tabular}{|c|c|c|c|c|}
\hline Microclimatic type & Abbreviation & Months & Avg. rainfall $(\mathrm{mm})$ & Avg. temperature $\left({ }^{\circ} \mathrm{C}\right)$ \\
\hline$\overline{\text { Dry }}$ & $\mathrm{D}$ & Jan-March & 4.07 & 33 \\
\hline Dry-wet & DW & April-June & 13.84 & 30.93 \\
\hline Wet & W & July-September & 19.94 & 27.90 \\
\hline Wet-dry & WD & September-Dec & 6 & 30.53 \\
\hline
\end{tabular}

Avg.: Average

Table 3: Statistical summary of the groundwater analysis for the different sampling period

\begin{tabular}{|c|c|c|c|c|c|c|c|c|}
\hline \multirow[b]{2}{*}{ Parameters } & \multicolumn{4}{|l|}{ Dry } & \multicolumn{4}{|c|}{ Dry-wet } \\
\hline & Min. & Max. & Mean & S.D. & Min. & Max. & Mean & S.D. \\
\hline$\overline{\mathrm{SWL}(\mathrm{m})}$ & 0.00 & 14.00 & 4.70 & 3.16 & 0.00 & 14.00 & 4.18 & 3.33 \\
\hline Temperature $\left({ }^{\circ} \mathrm{C}\right)$ & 27.80 & 32.20 & 28.80 & 0.93 & 27.80 & 32.00 & 29.34 & 1.15 \\
\hline Conductivity $(\mu \mathrm{sm} / \mathrm{cm})$ & 27.40 & 529.00 & 191.73 & 124.57 & 53.00 & 1141.00 & 310.00 & 296.32 \\
\hline $\mathrm{pH}$ & 6.00 & 9.10 & 7.33 & 0.73 & 5.30 & 9.10 & 7.34 & 1.03 \\
\hline Color (Pt-Co) & 0.00 & 51.00 & 6.86 & 15.05 & 0.00 & 825.00 & 76.18 & 210.46 \\
\hline $\mathrm{DO}(\mathrm{mg} / \mathrm{L})$ & 0.20 & 5.00 & 2.42 & 1.16 & 0.00 & 6.60 & 5.22 & 1.33 \\
\hline $\mathrm{BOD}(\mathrm{mg} / \mathrm{L})$ & 0.00 & 2.40 & 0.25 & 0.67 & 0.00 & 2.80 & 1.13 & 0.78 \\
\hline Total hardness & 18.00 & 2456.00 & 369.64 & 569.40 & 84.00 & 2188.00 & 653.82 & 554.59 \\
\hline Alkalinity (ppm) & 77.00 & 1090.00 & 364.77 & 316.95 & 40.00 & 870.00 & 216.82 & 222.74 \\
\hline $\mathrm{Na}^{+}(\mathrm{mg} / \mathrm{L})$ & 27.60 & 386.20 & 130.59 & 113.15 & 9.20 & 73.60 & 34.92 & 21.42 \\
\hline $\mathrm{K}^{+}(\mathrm{mg} / \mathrm{L})$ & 0.90 & 12.10 & 4.04 & 3.52 & 0.30 & 2.30 & 1.07 & 0.66 \\
\hline $\mathrm{Ca}^{2+}(\mathrm{mg} / \mathrm{L})$ & 5.60 & 199.60 & 82.85 & 63.43 & 14.40 & 347.90 & 125.25 & 93.22 \\
\hline $\mathrm{Mg}^{2+}(\mathrm{mg} / \mathrm{L})$ & 3.40 & 533.00 & 77.50 & 116.17 & 5.80 & 337.90 & 83.97 & 93.57 \\
\hline $\mathrm{NH}_{4}^{+}(\mathrm{mg} / \mathrm{L})$ & 0.09 & 1044.00 & 0.74 & 1.18 & 0.01 & 260.20 & 0.95 & 1.16 \\
\hline $\mathrm{HCO}_{3}^{-}(\mathrm{mg} / \mathrm{L})$ & 92.40 & 1308.00 & 437.73 & 380.30 & 48.00 & 1044.00 & 260.20 & 267.30 \\
\hline $\mathrm{Cl}^{-}(\mathrm{mg} / \mathrm{L})$ & 42.60 & 603.50 & 201.90 & 175.40 & 14.20 & 113.60 & 53.90 & 33.06 \\
\hline $\mathrm{SO}_{4}^{2-}(\mathrm{mg} / \mathrm{L})$ & 0.00 & 0.68 & 0.09 & 0.17 & 0.00 & 0.46 & 0.09 & 0.14 \\
\hline $\mathrm{NO}_{2}^{-}(\mathrm{mg} / \mathrm{L})$ & 0.00 & 8.00 & 0.23 & 0.43 & 0.00 & 0.90 & 0.07 & 0.19 \\
\hline $\mathrm{NO}_{3}^{-}(\mathrm{mg} / \mathrm{L})$ & 0.40 & 3.20 & 2.31 & 0.95 & 2.00 & 3.20 & 2.78 & 0.30 \\
\hline $\mathrm{PO}_{4}{ }^{3-}(\mathrm{mg} / \mathrm{L})$ & 0.05 & 0.80 & 0.22 & 0.21 & 0.04 & 1.85 & 0.26 & 0.39 \\
\hline Total Fe $(\mathrm{mg} / \mathrm{L})$ & 0.00 & 0.64 & 0.048 & 0.13 & 0.00 & 2.40 & 0.17 & 0.51 \\
\hline Total Mn (mg/L) & 0.00 & 24.00 & 5.06 & 6.38 & 0.04 & 11.40 & 2.47 & 3.69 \\
\hline $\mathrm{Zn}(\mathrm{mg} / \mathrm{L})$ & 0.00 & 0.00 & 0.00 & 0.00 & 0.00 & 0.09 & 0.0071 & 0.03 \\
\hline $\mathrm{THB}\left(\mathrm{x} 10^{3} \mathrm{cfu} / \mathrm{mL}\right)$ & 0.11 & 13.00 & 1.26 & 2.75 & 0.14 & 25.47 & 3.26 & 5.48 \\
\hline
\end{tabular}




\begin{tabular}{|c|c|c|c|c|c|c|c|c|}
\hline \multirow[b]{2}{*}{ Parameters } & \multicolumn{4}{|l|}{ Wet } & \multicolumn{4}{|c|}{ Wet-dry } \\
\hline & Min. & Max. & Mean & S.D. & Min. & Max. & Mean & S.D. \\
\hline Faecal coli $(/ 100 \mathrm{~mL})$ & 0.00 & 20.00 & 1.25 & 0.50 & 0.00 & 16.00 & 3.30 & 4.64 \\
\hline Total coli $(/ 100 \mathrm{~mL})$ & 0.00 & 15.00 & 5.56 & 3.97 & 0.00 & 134.00 & 14.93 & 33.55 \\
\hline SWL (m) & 0.00 & 13.20 & 2.97 & 3.27 & 0 & 7 & 2.74 & 2.00 \\
\hline Temperature $\left({ }^{\circ} \mathrm{C}\right)$ & 24.00 & 31.00 & 27.84 & 1.79 & 26.5 & 30 & 28.26 & 1.10 \\
\hline Conductivity $(\mu \mathrm{sm} / \mathrm{cm})$ & 33.00 & 1139.00 & 343.82 & 295.66 & 29 & 897 & 352.45 & 258.80 \\
\hline $\mathrm{pH}$ & 4.10 & 7.50 & 5.93 & 0.92 & 5.3 & 8.4 & 7.37 & 0.73 \\
\hline Color (Pt-Co) & 0.00 & 304.00 & 23.09 & 67.26 & 0 & 187 & 35.05 & 54.20 \\
\hline $\mathrm{DO}(\mathrm{mg} / \mathrm{L})$ & 0.00 & 5.60 & 3.83 & 1.31 & 1.2 & 5.2 & 3.57 & 1.10 \\
\hline $\mathrm{BOD}(\mathrm{mg} / \mathrm{L})$ & 0.00 & 0.80 & 0.04 & 0.29 & 0 & 2.4 & 0.25 & 0.60 \\
\hline Total hardness & 12.00 & 1258.00 & 417.54 & 357.69 & 12 & 318 & 105.82 & 78.70 \\
\hline Alkalinity (ppm) & 30.00 & 730.00 & 168.18 & 164.91 & 40 & 550 & 199.55 & 140.30 \\
\hline $\mathrm{Na}^{+}(\mathrm{mg} / \mathrm{L})$ & 13.80 & 73.60 & 29.83 & 18.63 & 13.8 & 69 & 30.95 & 16.40 \\
\hline $\mathrm{K}^{+}(\mathrm{mg} / \mathrm{L})$ & 0.40 & 2.30 & 0.92 & 0.56 & 0.4 & 2.1 & 0.96 & 0.51 \\
\hline $\mathrm{Ca}^{2+}(\mathrm{mg} / \mathrm{L})$ & 2.40 & 65.70 & 26.81 & 19.32 & 3.2 & 48.1 & 47.76 & 25.30 \\
\hline $\mathrm{Mg}^{2+}(\mathrm{mg} / \mathrm{L})$ & 7.30 & 272.40 & 88.15 & 77.50 & 0 & 48.1 & 13.52 & 14.50 \\
\hline $\mathrm{NH}_{4}^{+}(\mathrm{mg} / \mathrm{L})$ & 0.03 & 267.30 & 0.77 & 1.22 & 0 & 36 & 1.42 & 2.20 \\
\hline $\mathrm{HCO}_{3}^{-}(\mathrm{mg} / \mathrm{L})$ & 36.00 & 876.00 & 201.82 & 197.90 & 48 & 660 & 239.45 & 168.30 \\
\hline $\mathrm{Cl}^{-}(\mathrm{mg} / \mathrm{L})$ & 21.30 & 114.00 & 45.83 & 28.38 & 21.3 & 107 & 47.76 & 25.30 \\
\hline $\mathrm{SO}_{4}^{2-}(\mathrm{mg} / \mathrm{L})$ & 0.00 & 0.33 & 0.08 & 0.10 & 0 & 0.32 & 0.07 & 0.09 \\
\hline $\mathrm{NO}_{2}^{-}(\mathrm{mg} / \mathrm{L})$ & 0.00 & 1.40 & 0.10 & 0.30 & 0 & 1.4 & 0.12 & 0.30 \\
\hline $\mathrm{NO}_{3}^{-}(\mathrm{mg} / \mathrm{L})$ & 0.40 & 2.70 & 1.97 & 0.77 & 0.32 & 4.8 & 2.61 & 1.22 \\
\hline $\mathrm{PO}_{4}{ }^{3-}(\mathrm{mg} / \mathrm{L})$ & 0.00 & 2.41 & 0.42 & 0.60 & 0.03 & 0.83 & 0.26 & 0.26 \\
\hline Total Fe (mg/L) & 0.00 & 0.52 & 0.07 & 0.12 & 0 & 1.1 & 0.09 & 0.23 \\
\hline Total Mn (mg/L) & 0.21 & 12.70 & 5.93 & 4.36 & 0.1 & 11.9 & 3.56 & 3.42 \\
\hline $\mathrm{Zn}(\mathrm{mg} / \mathrm{L})$ & 0.00 & 0.14 & 0.01 & 0.15 & 0 & 0 & 0.00 & 0.00 \\
\hline $\mathrm{THB}\left(\times 10^{3} \mathrm{cfu} / \mathrm{mL}\right)$ & 0.00 & 6.10 & 1.48 & 1.86 & 0 & 21.1 & 2.85 & 4.50 \\
\hline Faecal coli $(/ 100 \mathrm{~mL})$ & 0.00 & 3.00 & 0.81 & 0.91 & 0 & 4 & 1.70 & 1.06 \\
\hline Total coli $(/ 100 \mathrm{~mL})$ & 0.00 & 20.00 & 5.09 & 5.94 & 0 & 31 & 9.15 & 9.80 \\
\hline
\end{tabular}

Min.: Minimum; Max.: Maximum; S.D.: Standard deviation

for heavy metals analysis were acidified to $\mathrm{pH}$ of 2 using nitric acid. The Physical parameters (Color, oxygen, $\mathrm{pH}$, conductivity and temperature) were determined in the field using standard field equipment.

\section{RESULTS}

Laboratory analysis: The groundwater samples were analyzed using standard methods. The statistical summary of the groundwater analysis for the different sampling period are given in Table 3 while Table 4 to 6 contain the mean values for the three lithologies.

Table 7 is the results of Soil Analysis in the study area while Table 8 are the factors and their ratings in vulnerability index of (SCONC).

\section{DISCUSSION}

Variation with formation: Static water level varies from 0 to $14 \mathrm{~m}$ with the lowest mean value of $1.56 \mathrm{~m}$ in Eze-Aku Formation and the highest mean value of 6.47 $\mathrm{m}$ in the intrusive. Temperature range from 24 to $32.2^{\circ} \mathrm{C}$ with the lowest mean value of $28.29^{\circ} \mathrm{C}$ in intrusive and highest mean value of $28.76^{\circ} \mathrm{C}$ in Eze-Aku Formation. The high temperature may be attributed to the physiographic condition of the area. Conductivity range from 27.4 to $1141 \mu \mathrm{s} / \mathrm{cm}$ with the lowest mean value of $185.34 \mu \mathrm{s} / \mathrm{cm}$ in intrusive and the highest mean value of $417.46 \mu \mathrm{s} / \mathrm{cm}$ in Eze-Aku Formation. pH varies from 4.1 to 9.1 with the lowest mean value of 6.86 in Asu-river group and the highest mean value of 7.25 in the intrusive. The low pH may be attributed to subsurface runoff and organic waste while the high $\mathrm{pH}$ may be due to a decrease in the dissolved solute. Color varies from 0 (Pt$\mathrm{Co}$ ) to 127 (Pt-Co) with the lowest mean of 25.50 (Pt$\mathrm{Co}$ ) in intrusive and the highest mean value of 39.5 (PtCo) in Asu River Group. Udom et al. (1998) attributed the high value of color to iron contamination in water. DO (Dissolved Oxygen) range from 0 to $6.6 \mathrm{mg} / \mathrm{L}$ with the lowest mean value of $3.15 \mathrm{mg} / \mathrm{L}$ in Eze-Aku Formation and the highest mean value of $4.31 \mathrm{mg} / \mathrm{L}$ in intrusive. BOD values range from 0.7 to $24 \mathrm{mg} / \mathrm{L}$ with the lowest mean value of $0.34 \mathrm{mg} / \mathrm{L}$ in Asu-River group and a high of $0.48 \mathrm{mg} / \mathrm{L}$ in Eze-Aku Formation. Total hardness varies from 12 to $2456 \mathrm{mg} / \mathrm{L}$ with the lowest mean value of $280.83 \mathrm{mg} / \mathrm{L}$ in intrusive and the highest mean value of $530.29 \mathrm{mg} / \mathrm{L}$ in Eze-Aku Formation. Alkalinity and total hardness are usually nearly equal in concentration (when they are both reported in $\mathrm{mg} / \mathrm{L}$ $\mathrm{CaCO}_{3}$ (calcium carbonate)), because they form from the same minerals (Spurlock, 2005). Alkalinity varies from 30 to $1090 \mathrm{mg} / \mathrm{L}$ with the lowest mean value of 164.13 $\mathrm{mg} / \mathrm{L}$ in intrusive and the highest mean value of $343.86 \mathrm{mg} / \mathrm{L}$ in Eze-Aku Formation. Sodium $\left(\mathrm{Na}^{+}\right)$ varies from a low of $9.2 \mathrm{mg} / \mathrm{L}$ to a high of $386.2 \mathrm{mg} / \mathrm{L}$ with the lowest mean value of $30.19 \mathrm{mg} / \mathrm{L}$ in intrusive and the highest mean value of $73.60 \mathrm{mg} / \mathrm{L}$ in Eze-Aku Formation. Higher concentrations of Sodium usually indicate contamination by septic systems, road salt, fertilizer, animal waste or other wastes. Potassium $\left(\mathrm{K}^{+}\right)$ 
Table 4: Mean values of parameters in intrusive

\begin{tabular}{|c|c|c|c|c|}
\hline Parameters & Dry & Dry-wet & Wet & Wet-dry \\
\hline s.w.1 (m) & 8.17 & 7.67 & 6.10 & 3.93 \\
\hline Temp. $\left({ }^{\circ} \mathrm{C}\right)$ & 29.17 & 28.67 & 27.08 & 28.23 \\
\hline Conductivity $(\mu \mathrm{sm} / \mathrm{cm})$ & 124.60 & 221.60 & 165.33 & 229.83 \\
\hline $\mathrm{pH}$ & 7.45 & 8.12 & 6.15 & 7.30 \\
\hline Color (Pt-Co) & 0.00 & 18.17 & 52.00 & 33.00 \\
\hline $\mathrm{DO}(\mathrm{mg} / \mathrm{L})$ & 2.67 & 5.68 & 4.78 & 4.10 \\
\hline $\mathrm{BOD}(\mathrm{mg} / \mathrm{L})$ & 0.00 & 0.95 & 0.23 & 0.22 \\
\hline Total hardness & 207.67 & 507.33 & 312.67 & 95.67 \\
\hline Alkalinity (ppm) & 169.83 & 178.33 & 118.33 & 190.00 \\
\hline $\mathrm{Na}^{+}(\mathrm{mg} / \mathrm{L})$ & 60.95 & 23.77 & 16.87 & 19.17 \\
\hline $\mathrm{k}^{+}(\mathrm{mg} / \mathrm{L})$ & 1.87 & 0.73 & 0.52 & 0.60 \\
\hline $\mathrm{Ca}^{2+}(\mathrm{mg} / \mathrm{L})$ & 61.57 & 78.55 & 17.63 & 14.82 \\
\hline $\mathrm{Mg}^{2+}(\mathrm{mg} / \mathrm{L})$ & 44.75 & 75.57 & 65.23 & 14.25 \\
\hline $\mathrm{NH}_{4}^{+}(\mathrm{mg} / \mathrm{L})$ & 0.17 & 0.48 & 0.33 & 0.63 \\
\hline $\mathrm{HCO}_{3}^{-}$ & 203.80 & 214.00 & 142.00 & 228.00 \\
\hline $\mathrm{Cl}^{-}(\mathrm{mg} / \mathrm{L})$ & 94.12 & 36.68 & 26.03 & 29.58 \\
\hline $\mathrm{SO}_{4}{ }^{2-}(\mathrm{mg} / \mathrm{L})$ & 0.00 & 0.00 & 0.02 & 0.02 \\
\hline $\mathrm{NO}_{2}^{-}(\mathrm{mg} / \mathrm{L})$ & 0.04 & 0.01 & 0.02 & 0.03 \\
\hline $\mathrm{NO}_{3}^{-}(\mathrm{mg} / \mathrm{L})$ & 2.03 & 2.93 & 1.78 & 1.98 \\
\hline $\mathrm{PO}_{4}{ }^{3-}(\mathrm{mg} / \mathrm{L})$ & 0.37 & 0.26 & 0.16 & 0.17 \\
\hline Total Fe (mg/L) & 0.01 & 0.09 & 0.08 & 0.05 \\
\hline Total Mn (mg/L) & 0.00 & 0.03 & 3.36 & 0.82 \\
\hline $\mathrm{Zn}(\mathrm{mg} / \mathrm{L})$ & 0.00 & 0.02 & 0.00 & 0.00 \\
\hline THB $\left(\times 10^{3} \mathrm{cfu} / \mathrm{mL}\right.$ & 2.54 & 1.31 & 1.30 & 1.95 \\
\hline Faecal coli $(/ 100 \mathrm{~mL})$ & 0.00 & 1.00 & 0.67 & 2.00 \\
\hline Total coli $(/ 100 \mathrm{~mL})$ & 2.50 & 3.40 & 3.17 & 5.25 \\
\hline$\frac{\text { 1 able 5: Mean values of }}{\text { Parameters }}$ & $\frac{1 n \text { Asu-r }}{\text { Dry }}$ & Dry wet & Wet & Wet-dry \\
\hline s.w.1 (m) & 4.73 & 4.29 & 3.19 & 3.54 \\
\hline Temp. $\left({ }^{\circ} \mathrm{C}\right)$ & 28.73 & 29.64 & 27.82 & 28.14 \\
\hline Conductivity $(\mu \mathrm{sm} / \mathrm{cm})$ & 192.46 & 285.81 & 337.56 & 321.78 \\
\hline $\mathrm{pH}$ & 7.33 & 6.91 & 5.87 & 7.32 \\
\hline Color (Pt-Co) & 8.33 & 74.78 & 14.44 & 60.44 \\
\hline $\mathrm{BOD}(\mathrm{mg} / \mathrm{L})$ & 0.33 & 0.96 & 0.02 & 0.06 \\
\hline $\mathrm{DO}(\mathrm{mg} / \mathrm{L})$ & 2.56 & 5.20 & 4.12 & 3.60 \\
\hline Total hardness & 486.89 & 413.78 & 397.11 & 84.67 \\
\hline Alkalinity (ppm) & 396.44 & 122.22 & 132.22 & 162.22 \\
\hline $\mathrm{Na}^{+}(\mathrm{mg} / \mathrm{L})$ & 142.11 & 36.29 & 33.04 & 32.20 \\
\hline $\mathrm{k}^{+}(\mathrm{mg} / \mathrm{L})$ & 4.38 & 1.12 & 1.02 & 0.99 \\
\hline $\mathrm{Ca}^{2+}(\mathrm{mg} / \mathrm{L})$ & 91.56 & 84.42 & 23.33 & 20.12 \\
\hline $\mathrm{Mg}^{2+}(\mathrm{mg} / \mathrm{L})$ & 99.24 & 52.11 & 82.30 & 8.78 \\
\hline $\mathrm{NH}_{4}^{+}(\mathrm{mg} / \mathrm{L})$ & 0.58 & 0.85 & 0.83 & 1.80 \\
\hline $\mathrm{HCO}_{3}^{-}$ & 396.44 & 146.67 & 158.67 & 194.67 \\
\hline $\mathrm{Cl}^{-}(\mathrm{mg} / \mathrm{L})$ & 219.33 & 56.01 & 50.49 & 49.70 \\
\hline $\mathrm{SO}_{4}^{2-}(\mathrm{mg} / \mathrm{L})$ & 0.10 & 0.06 & 0.08 & 0.06 \\
\hline $\mathrm{NO}_{2}^{-}(\mathrm{mg} / \mathrm{L})$ & 0.16 & 0.14 & 0.05 & 0.08 \\
\hline $\mathrm{NO}_{3}^{-}(\mathrm{mg} / \mathrm{L})$ & 2.13 & 2.89 & 2.11 & 2.53 \\
\hline $\mathrm{PO}_{4}{ }^{3-}(\mathrm{mg} / \mathrm{L})$ & 0.13 & 0.16 & 0.28 & 0.20 \\
\hline Total Fe (mg/L) & 0.03 & 0.07 & 0.05 & 0.05 \\
\hline Total Mn (mg/L) & 6.91 & 3.05 & 6.72 & 3.42 \\
\hline $\mathrm{Zn}(\mathrm{mg} / \mathrm{L})$ & 0.00 & 0.00 & 0.00 & 0.00 \\
\hline THB $\left(\times 10^{3} \mathrm{cfu} / \mathrm{mL}\right)$ & 0.68 & 2.14 & 1.29 & 4.00 \\
\hline Faecal coli $(/ 100 \mathrm{~mL})$ & 1.00 & 2.25 & 0.67 & 1.75 \\
\hline Total coli $(/ 100 \mathrm{~mL})$ & 5.00 & 9.80 & 5.00 & 9.11 \\
\hline $\begin{array}{l}\text { 1 able 6: M } \\
\text { Parameter }\end{array}$ & Dry & Dry-wet & Wet & Wet-dry \\
\hline s.w.1 (m) & 2.59 & 1.94 & 0.78 & 0.91 \\
\hline Temp. $\left({ }^{\circ} \mathrm{C}\right)$ & 28.57 & 29.53 & 28.50 & 28.43 \\
\hline Conductivity $(\mu \mathrm{sm} / \mathrm{cm})$ & 248.34 & 419.63 & 504.86 & 497.00 \\
\hline $\mathrm{pH}$ & 7.21 & 7.21 & 5.81 & 7.50 \\
\hline Color (Pt-Co) & 11.86 & 127.71 & 9.43 & 4.14 \\
\hline $\mathrm{DO}(\mathrm{mg} / \mathrm{L})$ & 2.03 & 4.86 & 2.63 & 3.07 \\
\hline $\mathrm{BOD}(\mathrm{mg} / \mathrm{L})$ & 0.00 & 1.50 & -0.11 & 0.53 \\
\hline Total hardness & 357.71 & 1088.00 & 533.71 & 141.71 \\
\hline Alkilinity (ppm) & 491.14 & 371.43 & 257.14 & 255.71 \\
\hline
\end{tabular}


Table 6: Continue

\begin{tabular}{|c|c|c|c|c|}
\hline Parameter & Dry & Dry-wet & Wet & Wet-dry \\
\hline $\mathrm{Na}^{+}(\mathrm{mg} / \mathrm{L})$ & 175.47 & 42.71 & 36.80 & 39.43 \\
\hline $\mathrm{k}^{+}(\mathrm{mg} / \mathrm{L})$ & 5.46 & 1.30 & 1.14 & 1.23 \\
\hline $\mathrm{Ca}^{2+}(\mathrm{mg} / \mathrm{L})$ & 89.91 & 217.79 & 39.16 & 26.13 \\
\hline $\mathrm{Mg}^{2+}(\mathrm{mg} / \mathrm{L})$ & 77.63 & 132.13 & 115.30 & 19.00 \\
\hline $\mathrm{NH}_{4}^{+}(\mathrm{mg} / \mathrm{L})$ & 1.43 & 1.48 & 1.08 & 1.60 \\
\hline $\mathrm{HCO}_{3}^{-}$ & 589.37 & 445.71 & 308.57 & 306.86 \\
\hline $\mathrm{Cl}^{-}(\mathrm{mg} / \mathrm{L})$ & 271.86 & 65.93 & 56.80 & 60.86 \\
\hline $\mathrm{SO}_{4}{ }^{2-}(\mathrm{mg} / \mathrm{L})$ & 0.17 & 0.19 & 0.14 & 0.11 \\
\hline $\mathrm{NO}_{2}^{-}(\mathrm{mg} / \mathrm{L})$ & 0.47 & 0.02 & 0.23 & 0.26 \\
\hline $\mathrm{NO}_{3}^{-}(\mathrm{mg} / \mathrm{L})$ & 2.79 & 2.51 & 1.96 & 3.14 \\
\hline $\mathrm{PO}_{4}{ }^{3-}(\mathrm{mg} / \mathrm{L})$ & 0.22 & 0.40 & 0.82 & 0.41 \\
\hline Total Fe $(\mathrm{mg} / \mathrm{L})$ & 0.11 & 0.36 & 0.10 & 0.20 \\
\hline Total Mn (mg/L) & 7.01 & 3.83 & 7.13 & 6.07 \\
\hline $\mathrm{Zn}(\mathrm{mg} / \mathrm{L})$ & 0.00 & 0.00 & 0.00 & 0.00 \\
\hline THB $\left(\times 10^{3} \mathrm{cfu} / \mathrm{mL}\right.$ & 0.90 & 6.38 & 1.89 & 2.31 \\
\hline Faecal coli $(/ 100 \mathrm{~mL})$ & 2.00 & 5.50 & 1.14 & 1.60 \\
\hline Total coli $(/ 100 \mathrm{~mL})$ & 15.00 & 31.60 & 6.86 & 11.43 \\
\hline
\end{tabular}

Table 7: The result of soil analysis in the study area

\begin{tabular}{|c|c|c|c|c|c|c|c|c|c|}
\hline Locations & $\mathrm{a}$ & $\mathrm{b}$ & $\mathrm{c}$ & $\mathrm{d}$ & $\mathrm{e}$ & $\mathrm{f}$ & $\mathrm{g}$ & Mean & S.D. \\
\hline Sand $(\mathrm{g} / \mathrm{kg})$ & 516 & 546 & 796 & 866 & 836 & 816 & 826 & 743 & 135.80 \\
\hline Silt (g/kg) & 237 & 147 & 177 & 127 & 87 & 177 & 147 & 157 & 43.40 \\
\hline Clay (g/kg) & 244 & 304 & 27 & 7 & 77 & 7 & 27 & 99 & 113.90 \\
\hline Texture & SCL & SCL & LS & LS & LS & LS & LS & & \\
\hline $\mathrm{pH}$ & 5.01 & 4.82 & 4.51 & 4.92 & 4.81 & 4.62 & 5.03 & 4.81 & 0.18 \\
\hline $\mathrm{OC} \%$ & 1.58 & 2.21 & 1.06 & 0.92 & 0.81 & 1.01 & 1.20 & 1.25 & 0.45 \\
\hline Org. M \% & 2.72 & 3.81 & 1.82 & 1.58 & 1.39 & 1.74 & 2.06 & 2.16 & 0.78 \\
\hline $\mathrm{N} \%$ & 1.00 & 1.03 & 0.09 & 0.85 & 1.13 & 1.11 & 1.21 & 0.92 & 0.35 \\
\hline $\mathrm{P}(\mathrm{mg} / \mathrm{kg})$ & 14.00 & 12.00 & 9.00 & 5.00 & 8.00 & 6.00 & 11.00 & 9.28 & 3.01 \\
\hline $\mathrm{K}(\mathrm{cmol} / \mathrm{kg})$ & 0.33 & 0.24 & 0.19 & 0.08 & 0.22 & 0.14 & 0.21 & 0.20 & 0.07 \\
\hline $\mathrm{Ca}(\mathrm{cmol} / \mathrm{kg})$ & 2.41 & 2.02 & 1.20 & 1.20 & 0.90 & 1.50 & 0.70 & 1.42 & 0.56 \\
\hline $\mathrm{Mg}(\mathrm{cmol} / \mathrm{kg})$ & 2.30 & 1.80 & 0.33 & 0.58 & 0.20 & 0.21 & 0.54 & 0.85 & 0.78 \\
\hline $\mathrm{Na}(\mathrm{cmol} / \mathrm{kg})$ & 0.10 & 0.09 & 0.11 & 0.06 & 0.10 & 0.05 & 0.10 & 0.08 & 0.02 \\
\hline $\mathrm{H}+\mathrm{Al}(\mathrm{cmol} / \mathrm{kg})$ & 1.60 & 1.72 & 1.01 & 1.21 & 1.31 & 0.91 & 0.98 & 1.25 & 0.29 \\
\hline $\mathrm{ECEC}(\mathrm{cmol} / \mathrm{kg})$ & 6.74 & 5.87 & 2.84 & 3.13 & 2.73 & 2.81 & 2.53 & 3.81 & 1.60 \\
\hline$\underline{\mathrm{BS} \%}$ & 76.28 & 70.70 & 64.43 & 61.34 & 52.01 & 67.61 & 61.26 & 64.80 & 7.78 \\
\hline
\end{tabular}

a, b: Intrusive; c, d, e: Asu river group; f, g: Eze-Aku formation; SCL: Sandy clay loamy; LS: Loamy sand; S.D.: Standard deviation

Table 8: Factors and their ratings in vulnerability index of (sconc)

\begin{tabular}{llll}
\hline Factor & Weight $(w)$ & $\begin{array}{l}\text { Range of } \\
\text { rating }\end{array}$ & $\begin{array}{l}\text { Parameter } \\
\text { range }\end{array}$ \\
\hline Static water table & 5 & $1-5$ & $0-14$ \\
$\mathrm{CEC}$ & 4 & $1-5$ & $0-10$ \\
$\mathrm{OC}$ & 3 & $1-5$ & $0-2.5$ \\
$\mathrm{NO}_{3}^{-}$ & 2 & $1-5$ & $10-650$ \\
$\mathrm{CI}^{-}$ & 1 & $1-5$ & $0-10$ \\
\hline
\end{tabular}

range from 0.3 to $12.1 \mathrm{mg} / \mathrm{L}$ with the lowest mean value of $0.93 \mathrm{mg} / \mathrm{L}$ in intrusive and the highest mean value of $2.28 \mathrm{mg} / \mathrm{L}$ in Eze-Aku Formation. Calcium $\left(\mathrm{Ca}^{2+}\right)$ range from 2.4 to $347.9 \mathrm{mg} / \mathrm{L}$ with the lowest mean value of $43.14 \mathrm{mg} / \mathrm{L}$ in intrusive and the highest mean value of $93.25 \mathrm{mg} / \mathrm{L}$ in Eze-Aku Formation. Magnesium $\left(\mathrm{Mg}^{2+}\right)$ varies from 0 to $534 \mathrm{mg} / \mathrm{L}$ with the lowest mean value of $49.95 \mathrm{mg} / \mathrm{L}$ in intrusive and the highest mean value of $86.02 \mathrm{mg}$ in Eze-Aku Formation. Most concentrations are higher than the WHO values of $100 \mathrm{mg} / \mathrm{L}$ for Calcium and $50 \mathrm{mg} / \mathrm{L}$ for Magnesium. As water moves through soil and rock, it dissolves very small amounts of minerals and holds them in solution. Calcium and magnesium dissolved in water are the two most common minerals that make water "hard." The degree of hardness becomes greater as the calcium and magnesium content increases and is related to the concentration of multivalent cations dissolved in the water. Ammonium $\left(\mathrm{NH}_{4}{ }^{+}\right)$range from 0.01 to $10.7 \mathrm{mg} / \mathrm{L}$ with the lowest mean value of $0.40 \mathrm{mg} / \mathrm{L}$ in intrusive and the highest mean value of $1.39 \mathrm{mg} / \mathrm{L}$ in Eze-Aku Formation. Bicarbonate $\left(\mathrm{HCO}_{3}{ }^{-}\right)$range from 36 to $1308 \mathrm{mg} / \mathrm{L}$ with the lowest mean value of $196.95 \mathrm{mg} / \mathrm{L}$ in intrusive and the highest mean value of $412.63 \mathrm{mg} / \mathrm{L}$ in Eze-Aku Formation. Chloride $\left(\mathrm{Cl}^{-}\right)$varies from 14.2 to 603.5 $\mathrm{mg} / \mathrm{L}$ with the lowest mean value of $46.60 \mathrm{mg} / \mathrm{L}$ in intrusive and the highest mean value of $113.86 \mathrm{mg} / \mathrm{L}$ in Eze-Aku Formation. The high concentration may be due to contamination by septic systems, road salt, fertilizer, animal waste, landfills, or other wastes. Chlorides are salts resulting from the combination of the gas chlorine with a metal. Some common chlorides include sodium chloride $(\mathrm{NaCl})$ and magnesium chloride $\left(\mathrm{MgCl}_{2}\right)$. Chlorine alone as $\mathrm{Cl}_{2}$ is highly toxic and it is often used as a disinfectant. In combination with a metal such as sodium it becomes essential for life. Small amounts of chlorides are required for normal cell functions in plant and animal life. Chlorides are not usually harmful to people; however, the sodium part of table salt has been linked to heart and kidney disease. Sodium chloride may impart a salty taste at $250 \mathrm{mg} / \mathrm{L}$; however, calcium or magnesium chlorides are not usually detected by taste until levels of $1000 \mathrm{mg} / \mathrm{L}$ are reached. The amount of chlorine that reacts with the other chemicals plus the amount required to achieve disinfection is the chlorine 
demand of the water. Chlorides may get into surface water from several sources including: rocks containing chlorides; agricultural runoff; wastewater from industries; oil well wastes; effluent wastewater from wastewater treatment plants and; road salting. Chlorides can corrode metals and affect the taste of food products. Therefore, water that is used in industry or processed for any use has a recommended maximum chloride level. Chlorides can contaminate freshwater streams and lakes. Fish and aquatic communities cannot survive in high levels of chlorides (Farrar, 1997). Nitrate $\left(\mathrm{NO}_{3}{ }^{-}\right)$range from 0.4 to $4.8 \mathrm{mg} / \mathrm{L}$ with the lowest mean value of 2.18 $\mathrm{mg} / \mathrm{L}$ in intrusive and the highest mean value of 2.60 $\mathrm{mg} / \mathrm{L}$ in Eze-Aku Formation. Sulphate $\left(\mathrm{SO}_{4}{ }^{2+}\right)$ range from 0 to $0.68 \mathrm{mg} / \mathrm{L}$ with the lowest mean value of 0.01 $\mathrm{mg} / \mathrm{L}$ in intrusive and the highest mean value of 0.15 $\mathrm{mg} / \mathrm{L}$ in Eze-Aku Formation. Nitrate $\left(\mathrm{NO}_{3}{ }^{-}\right)$is a common inorganic form of nitrogen. Chemically, it is an anion with a single negative charge, consisting of one atom of nitrogen and three atoms of oxygen. Because it is an anion, it is soluble in water. Plants normally use nitrate as their source of the nitrogen needed by all living things and so nitrate is considered a nutrient for plants. Excessive concentrations of nitrate in lakes and streams greater than about $5 \mathrm{mg} / \mathrm{L}$ (measured as nitrogen), depending on the water body, can cause excessive growth of algae and other plants, leading to accelerated eutrophication or "aging" of lakes and occasional loss of dissolved oxygen. If nitrate-nitrogen exceeds $10 \mathrm{mg} / \mathrm{L}$ in drinking water, it can cause a condition called methemoglobinemia or "blue baby syndrome" in infants (Fan et al., 1987). Some studies has indicated a possible connection between elevated nitrate concentrations and cancer (US Geological Survey, 2004). Nitride $\left(\mathrm{NO}_{2}^{-}\right)$ varies from 0 to $1.5 \mathrm{mg} / \mathrm{L}$ with the lowest mean value of $0.02 \mathrm{mg} / \mathrm{L}$ in intrusive and the highest mean value of $0.24 \mathrm{mg} / \mathrm{L}$ in Eze-Aku Formation. High concentration of nitrite may be due to waste. Phosphate $\left(\mathrm{PO}_{4}{ }^{3-}\right)$ varies from 0.03 to $2.41 \mathrm{mg} / \mathrm{L}$ with the lowest mean value of $0.19 \mathrm{mg} / \mathrm{L}$ in Asu-River group and the highest mean value of $0.46 \mathrm{mg} / \mathrm{L}$ in Eze-Aku Formation. Sulphate $\left(\mathrm{SO}_{4}{ }^{2-}\right)$ and Phosphate $\left(\mathrm{PO}_{4}{ }^{3-}\right)$ are within WHO (2001) standard values, Phosphates are not toxic to people or animals unless they are present in very high levels. Digestive problems could occur from extremely high levels of phosphate (D'Angelo et al., 2001). Total Iron $(\mathrm{mg} / \mathrm{L})$ range from 0 to $2.4 \mathrm{mg} / \mathrm{L}$ with the lowest mean value of $0.05 \mathrm{mg} / \mathrm{L}$ in Asu-River group and the highest mean level of $0.19 \mathrm{mg} / \mathrm{L}$ in Eze-Aku Formation. Zinc ( $\mathrm{Zn})$ varies from 0 to $0.14 \mathrm{mg} / \mathrm{L}$ with the lowest mean value of $0 \mathrm{mg} / \mathrm{L}$ in Asu-River group and the highest mean value of $0.02 \mathrm{mg} / \mathrm{L}$ in intrusive. Iron $(\mathrm{Fe})$, Total Manganese $(\mathrm{mg} / \mathrm{L})$ values were higher than the WHO (2001) values, while Zinc ( $\mathrm{Zn}$ ) was below WHO value. Dissolved ferrous iron gives water a disagreeable taste. When the iron combines with tea, coffee and other beverages, it produces an inky, black appearance and a harsh, unacceptable taste. Manganese is too reactive a metal to be found in elemental form in nature but its ore, the black dioxide pyrolusite, is widely distributed. The high concentration of metals in groundwater may be attributed to biodegradation of organic wastes which may lead to the release of these metals which are in turn leached into the water by the process of desorption. Total Manganese (mg/L) range from 0 to $24 \mathrm{mg} / \mathrm{L}$ with the lowest mean concentration of $1.05 \mathrm{mg} / \mathrm{L}$ in intrusive and the highest mean concentration of $6.01 \mathrm{mg} / \mathrm{L}$ in Eze-Aku Formation, most other element follow the same pattern exception of $\mathrm{PO}_{4}{ }^{3-}, \mathrm{Fe}$ and $\mathrm{Zn}$ which had their low concentration in Asu-River group and high concentration in Eze-Aku Formation. Total Heterotrophic Bacteria (THB) range from 0 to 25.47 $\mathrm{cfu} / \mathrm{mL}$ with the lowest mean value of $1.78 \mathrm{cfu} / \mathrm{mL}$ in intrusive and the highest mean value of $2.87 \mathrm{cfu} / \mathrm{mL}$ in Eze-Aku Formation. The presence of fecal coliform in drinking water is evidence that human or animal waste has been or is present. This may be cause for concern because many diseases can be spread through fecal transmission. Escherichia coli, is a specific type of fecal coliform, the coliform group that lives in the intestines of humans and other warm-blooded animals and in their waste. Human illnesses such as typhoid, dysentery, cholera, hepatitis and giardiasis have been linked to drinking water contaminated by human waste (Brunett et al., 1997). Faecal coliform varies from 0 to $16 \mathrm{cfu} / \mathrm{mL}$ with the lowest mean value of $0.92 \mathrm{cfu} / \mathrm{mL}$ in intrusive and the highest mean value of $2.56 \mathrm{cfu} / \mathrm{mL}$ in Eze-Aku Formation. Total Coliform bacteria are live organisms and they can multiply rapidly or die off quickly, depending upon water temperature and other variables like Chlorine and Iodine which kills bacteria, including disease-causing organisms and the nuisance organism, iron bacteria. This naturally occurring bacterium does not cause disease, but does form a reddish brown slime that coats the inside of pipes, fouls pumps and clogs waters (Dorsch et al., 1984). Coliform range from 2 to $134 \mathrm{cfu} / \mathrm{mL}$ with the lowest mean value of $3.58 \mathrm{cfu} / \mathrm{mL}$ in intrusive and the highest mean value of $16.22 \mathrm{cfu} / \mathrm{mL}$ in Eze-Aku Formation.

Vulnerability modeling: A commonly used model in assessing groundwater vulnerability is the DRASTIC model (Aller et al., 1985; Deichert and Hamlet, 1992; Aller et al., 1987; Kim and Hamm, 1999). The parameters of DRASTIC are weighted according to their relative importance in determining the ability of a pollutant to reach an aquifer.

In this study, Static water level, Cation Exchange capacity, Organic content, Nitrate and Chlorine (SCONC) are used to determine the vulnerability of the different Formation. SCONC index was calculated using the following formulae:

SCONC index $=\mathrm{S}_{\mathrm{r}} \mathrm{S}_{\mathrm{w}}+\mathrm{C}_{\mathrm{r}} \mathrm{C}_{\mathrm{w}}+\mathrm{O}_{\mathrm{r}} \mathrm{O}_{\mathrm{w}}+\mathrm{N}_{\mathrm{r}} \mathrm{N}_{\mathrm{w}}+\mathrm{C}_{\mathrm{r}} \mathrm{C}_{\mathrm{w}}$ 
Table 9: Vulnerability indices of SCONC $=$ Index in the different formations

\begin{tabular}{llll}
\hline Values & Intrusive & Asu-river group & $\begin{array}{l}\text { Eze-Aku } \\
\text { formation }\end{array}$ \\
\hline & $(2) 43$ & $(5) 43$ & $(9) 46$ \\
& $(7) 53$ & $(13) 34$ & $(10) 49$ \\
& $(18) 52$ & $(15) 39$ & $(11) 43$ \\
& & $(16) 46$ & $(12) 43$ \\
& & $(19) 37$ & $(17) 47$ \\
Min. & 43 & $(20) 34$ & \\
Max. & 53 & $(21) 40$ & 43 \\
Mean & 49.30 & 44 & 49 \\
S.D. & 5.51 & 39 & 45.60 \\
\hline Min: & & 4.47 & 2.61 \\
\hline
\end{tabular}

Min.: Minimum; Max.: Maximum; S.D.: Standard deviation

where,

$\mathrm{r} \quad=$ Rating for the area being evaluated (1-5)

$\mathrm{w}=$ Importance weight for the factor (1-5)

Factor ratings are derived from data on each factor while importance weights are found in a generic DRASTIC table that lists weights for factors having greater applicability (Aller et al., 1987). The Sconc index was further divided into 4 categories: low, moderate, high and very high. Low (10-20), moderate (20-40), high (40-60), very high $>60$. From Table 9, Asu-River group is moderately vulnerable while Intrusive and Eze-Aku Formation is said to be highly vulnerable to pollution and this may be due to fracture in the intrusive which allows free flow of water into the water table while that of EzeAku Formation may be due to shallow depth of the water table to the surface.

\section{CONCLUSION}

Baseline data on hydro geochemical studies and vulnerability assessment of sandstone-shale-intrusive aquifers which previously had not been documented have been assembled and analyzed in this study. The present levels of physicochemical parameters, biological, heavy metal have been used to assess the vulnerability of sandstone-shale-intrusive aquifer in the study area. In physiochemical parameters, more than $70 \%$ of the parameters are below the W.H.O standard value for drinking and domestic purposes. More than $65 \%$ of biological parameters are below W.H.O standard while the other percentage of more than $25 \%$ is higher than W.H.O standard and was noticed to increase during a wet and wet-dry period and reduces during a dry period which shows an increase due to infiltration. It was also noticed to increase from Asu River group to Eze-Aku Formation. More than $40 \%$ of the heavy metal was higher than the W.H.O standard values. The data obtained show that contamination or increase in parameters follows the flow direction of groundwater. It was also observed that the Eze-Aku Formation was more vulnerable to coliform than the Asu-River Group followed by the Intrusive. The data also show that the formations were more vulnerable in the wet period in some area than dry period while in some area the wet period reduces the vulnerability due to dilution from runoff and this is evidence that the vulnerability of the study area is control by infiltration and runoff. And these go further to show that the aquifer in the study area is mostly recharged by precipitation. The data also show that the aquifers in the study area are not vulnerable to nitrate but are rather vulnerable to fecal coliform due to water from runoff that infiltrates into the aquifer from the vadose zone during precipitation in the area. The result of vulnerability of the formations using SCONC index (Table 9) shows a moderate vulnerability of Asu-River group while Intrusive and Eze-Aku Formation is said to be highly vulnerable to pollution and this may be due to fracture in the intrusive which allows free flow of water into the water table while that of Eze-Aku Formation may be due to shallow depth of the water table to the surface.

\section{REFERENCES}

Aller, L., T. Bennett, J. Lehr and R. Petty, 1985. DRASTIC-A A standardized system for evaluating ground water pollution potential using hydrogeologic settings. US Environmental Protection Agency Report EPA/600/2-85/018, 163.

Aller, L., T. Bennet, J. Lehr, R. Petty and G. Hackett, 1987. DRASTIC; A standardized system for evaluating groundwater pollution potential using hydrogeologic settings. US Environmental Protection Agency Report, EPA600/2-87-035, 622.

Bartarya, S.K., 1993. Hydrochemistry and rock weathering in a sub-tropical lesser Himalayan river basin in Kumaun, India. J. Hydrol., 146: 149-174.

Brunett, J.O., N.L. Barber, A.W. Burns, R.P. Fogelman, D.C. Gillies, R.A. Lidwin and T.J. Mack, 1997. A quality-assurance plan for district ground-water activities of the U.S. Geological Survey. US Geological Survey Open-File Report 97-11, 21.

CRBDA (Cross River Basin Development Authority), 1982. Inventory of natural site conditions, soil slopes, hydrology, land use and vegetation through the area of operation of the authority. Cross River Basin Development Authority Progress Report, 4: 1-154.

D'Angelo, E., J. Crutchfield and M. Vandiviere, 2001. Rapid, sensitive, microscale determination of phosphate in water and soil. J. Environ. Qual., 30(6): 2206-2209.

Deichert, L.A. and J.M. Hamlet, 1992. Non-point groundwater pollution the potential in Pennsylvania. Proceeding of American Society of Agricultural Engineers (ASAE) International Winter Meeting, Nashville, Tennessee, December 15-18, 1992: Paper No. 92253. 
Dorsch, M.M., R.K. Scragg, A.J. McMichael, P.A. Baghurst and K.F. Dyer, 1984. Congenital malformations and maternal drinking water supply in rural South Australia: A case-control study. Am. J. Epidemiol., 119(04): 473-486.

Fan, A.M., C.C. Wilhite and S.A. Book, 1987. Evaluation of the nitrate drinking water standard with reference to infant methemoglobinemia and potential reproductive toxicity. Regul. Toxicol. Pharm., 7(02): 135-148.

Farrar, J.W., 1997. Results of the US geological survey's analytical evaluation program for standard reference sample. US Geological Survey Open-File Report 97-553, 184.

Freeze, R.A. and J.A. Cherry, 1979. Groundwater. Prentice-Hall, Englwood Cliffs, N.J., pp: 604.

Hearne, G.A., M. Wireman, A. Campbell, S. Turner and G.P. Ingersoll, 1992. Vulnerability of the uppermost ground water to contamination in the Greater Denver area, Colorado. US Geological Survey Water-Resources Investigations Report 92-4143, 243.

Iloeje, N.P., 1991. A New Geography of West Africa. 9th Edn., Nigeria.

Jacks, G., 1973. Chemistry of ground water in a district in Southern India. J. Hydrol., 18: 185-200.

Kim, Y.J. and S.Y. Hamm, 1999. Assessment of the potential for groundwater contamination using the DRASTIC/EGIS technique, Cheongju area, South Korea. Hydrogeol. J., 7(2): 227-235.

Matthess, G., 1982. The Properties of Groundwater. John Wiley, New York.

National Research Council, 1993. Groundwater Vulnerability Assessment, Contamination Potential under Conditions of Uncertainty. National Academy Press, Washington.
Petters, S.W., 1989. A regional hydrogeological study of rural Water supply options for planning and implementation of phase II rural water programmed in Cross River State (final volume). DFRRI, Cross River State Unpublished Report, 97.

Petters, S.W., C.S. Okereke and C.S. Nwajide, 1987. Geology of the Mamfe Rift, S. E. Nigeria. In: Matheis, G. and H. Schandelmeier, (Eds.), Current Research in African Earth Sciences, Balkema, Rotterdam, 1987, pp: 299-302.

Spurlock, D., 2005. Calcium and water quality. Natural Resources and Environmental Protection Cabinet Indiana University Southeast Indiana.

Udom, G.J., E.O. Esu and S.J. Ekwere, 1998. Quality status of groundwater in Calabar municipality, Southeastern Nigeria. Global J. Pure Appl. Sci., 4(2): 163-169.

US Geological Survey, 2004. Frequently Asked Questions: Why are High Nitrate or Nitrogen Concentrations in Water a Problem and What can be Done to Maintain Safe Levels? US Geological Survey Water-Supply Paper EPA816-R-004, 117.

Vowinkel, E.F., R.M. Clawges, D.E. Buxton, D.A. Stedfast and J.B. Louis, 1996. Vulnerability of public drinking water supplies in New Jersey to pesticides. US Geological Survey Fact Sheet FS165-96, 3.

WHO, 2001. Guidelines for Drinking Water Quality. World Health Organization Guidelines Publications, Geneva. 